\title{
Effect of the molecular weight on the depth profiling of PMMA thin films using low energy $\mathrm{Cs}^{+}$sputtering
}

\author{
Amal Ben Hadj Mabrouk ${ }^{1}$, Christophe Licitra ${ }^{1}$, Antoine Chateauminois ${ }^{2}$ and Marc Veillerot ${ }^{1}$ \\ ${ }^{1}$ Université Grenoble Alpes, CEA, Leti, F-38000 Grenoble, France \\ ${ }^{2}$ Soft Matter Science and Engineering Laboratory (SIMM), PSL Research University, \\ UPMC Univ. Paris 06, ESPCI Paris, CNRS, 10 rue Vauquelin, 75231 Paris Cedex 05, France
}

E-mail: marc.veillerot@cea.fr

\begin{abstract}
In this work, we investigate the influence of the molecular weight of poly (methyl methacrylate) (PMMA) thin films coated on silicon wafer on the ToF SIMS (Time of Flight Secondary Ion Mass Spectrometry) sputtering mechanisms and kinetics during depth profiling using low energy monoatomic caesium ions. The sputtering yield volumes are determined as function of molecular weight, film thickness and beam energy. The results show that the sputtering yield volume decreases with increasing molecular weight $M_{w}$ down to a threshold value below which it becomes nearly constant, as previously observed with argon cluster ions. The relevance of physical parameters such as the glass transition temperature $T_{g}$ - determined here from ellipsometry measurements - and the entanglement of the polymer chains to account for this behaviour is discussed. The variation of the sputtering yield was also found to vary logarithmically with the primary beam energy. In addition, preliminary experiments carried out using a low molecular weight PMMA (4 kg/mol) evidenced a nano-confinement effect similar to that observed with argon cluster sputtering but of lower magnitude.
\end{abstract}

Keywords: ToF-SIMS- Polymers- Molecular Weight- Caesium- Energy- Depth Profiling. 


\section{Introduction}

Owing to their unique mechanical and optical properties, polymeric materials are becoming widely used in the field of micro and nanotechnologies, for nano-impression and lithography applications or organic light emitting diodes, as an example. Their physical properties are controlled by polymer structural parameters such as molecular weight, polydispersity.... As the polymer surface's properties are basically governed by the molecular structure of the outermost layers, sensitive and depth-resolved analysis techniques are required for the design and development of such molecular systems for microelectronics. Within this context, ToF SIMS characterisation technique emerged as a powerful tool to investigate the near-surface structures ${ }^{1}$ and chemical composition ${ }^{2}$ of polymers. One of the limits of polymer chemical depth profiling using ToF-SIMS arises from the chemical damage induced by the formation of free radicals leading to chain scissions and crosslinking. However, since the introduction of polyatomic ion sources, depth profiling with ToF-SIMS is increasingly used for polymers. With polyatomic ions, the energy per bombarding atom is low (in the range of a few eV per atom for a cluster source with a few keV energy) and close to the dissociation energy of the polymer chemical bonds ${ }^{3,4}$ which reduces the depth of the altered region and damage accumulation, thus preserving the molecular information ${ }^{5}$. The use of innovative argon Gas Clusters Ions Beams (GCIB) have proven to be one of the most efficient approach in soft etching and sputtering of fragile organic materials, the depth resolution of the analysis ${ }^{3,5,6}$ being improved by the reduction of the degradation and fragmentation of polymer chains.

Recently, Cristaudo ${ }^{4}$ et al published an investigation of the effects of the molecular weight of two linear amorphous polymers, Poly-methyl-methacrylate (PMMA) and Polystyrene (PS) deposited on silicon substrates, on the efficiency of the sputtering process using $10 \mathrm{keV}$ argon clusters. This work showed that the sputtering yield volume is highly dependent on the molecular weight below a threshold value and then becomes constant for higher molecular weights. This behaviour was attributed by the authors to the variation of the glass transition $\left(T_{g}\right)$ of the polymers with $M_{w}$. Seah $e t a l^{7}$ showed afterwards that these data are fully consistent with the so-called universal sputtering equation ${ }^{8}$, an empirical equation describing the dependence of sputtering yield by argon clusters on energy, cluster size and materials chemical structure. This equation proved to be accurate for different types of organic (polystyrene (PS), polycarbonate (PC) and PMMA) and inorganic materials. More specifically, Seah et $a l^{7}$ ended by discussing the effects of molecular weight on the empirical parameter which reflects, in this equation, the bonding energy per atom required to eject the sputtered fragments. Seah et al attributed the dependency of this parameter on $M_{w}$ to the contribution of end groups to the sputtering process. 
The objective of the present work is to evaluate the ability of monoatomic caesium ions to improve the sensitivity of ToF-SIMS analysis to structural parameters of polymer thin films, remembering that the high sputtering yield induced by argon cluster ions ${ }^{9}$ sputtering is a limiting factor when it comes to nanometric thin films depth profiling.

When monoatomic primary ions penetrate the polymer with an energy ranging from hundreds to thousands of $\mathrm{eV}$, an energy transfer occurs via recoil collision cascades which directly leads to sputtering and species ejection ${ }^{10,11}$. The associated damage is further enhanced by the electronic excitations induced by the primary ions charge which results in fewer molecular ions and thus, in a limited access to a molecular information when depth profiling is carried out. However, investigations by Houssiau et $a l^{12}$.Different polymers (polycarbonate, polystyrene or polymethacrylate) sputtering processes by low-energy caesium primary ions have demonstrated that molecular depth-profiling with monoatomic ions can be successfully performed on polymers while maintaining their chemical structure and fingerprint.

According to a second study by Houssiau et $a l^{13}$ that aimed to explain the mechanisms involved in the sputtering process of organic materials, molecular depth profiling using monoatomic $\mathrm{Cs}^{+}$ion is relevant for three main reasons. ( $i$ ) the lower the impact energy, the lower the damage it generates within the polymer, (ii) the formation and emission of anions is enhanced due to the electron exchange between the alkali and polymer fragments, (iii) the reaction between free radicals and implanted neutralized $\mathrm{Cs}^{\circ}$ prevents the crosslinking and chain degradations and preserves the molecular information ${ }^{13}$.

Furthermore, it is known that the quality of a depth profile is controlled by operating parameters such as primary ions energy, angle of incidence, experimental temperature and sample's topography. Previous findings ${ }^{14,15}$ stated that projectile parameters including energy, affect directly the physical and chemical processes taking place upon ion bombardment. The primary ions' energy defines the induced damage accumulation ${ }^{16,17}$ and thus the sputtering yields.

In this study, we investigate the effects of molecular weight and confinement on the sputtering process of Poly(methyl methacrylate) (PMMA) thin films by monoatomic $\mathrm{Cs}^{+}$ions. For that purpose, we especially focus on the sputtering yield of thin PMMA films with varying molecular weight (in the 4-1260 kg/mol range) and thicknesses (in the 90-180 nm range) as a function of the beam energy. These results will be compared with GCIB sputtering as studied by Cristaudo et $a l^{4}$. 


\section{Experimental}

\section{Materials and sample preparation}

PMMA polymer pellets and powder with average molecular weights ranging from 4 to $1260 \mathrm{~kg} / \mathrm{mol}$ were purchased from Sigma Aldrich Inc. and Polymer Source Inc. The polydispersity of the polymers provided by Sigma Aldrich Inc. were measured using Size Exclusion Chromatography analysis, since the purchaser did not provide them. The molecular weights and polydispersity indexes of all the used PMMA are given in Table 1. The polymers have been used without any further purification. They were dissolved in Toluene (Sigma Aldrich, purity: >99.71\%) and spin-coated on pre-treated $\left(1 \times 1 \mathrm{~cm}^{2}\right)$ silicon substrates. Prior to use, the silicon substrates were successively cleaned in sulphuric and hydrofluoric acid and subsequently rinsed in deionized water. Then, Si samples were stored in plastic boxes until PMMA film deposition. Prior to spin-coating, silicon substrates were systematically sonicated in acetone and dried under $\mathrm{N}_{2}$ flow. They were first spin-coated with pure solvent in order to enhance the wettability of the substrate by the polymer solution. The concentration of, rotational speed and acceleration were adjusted in order to achieve film thicknesses in the range $90-180 \mathrm{~nm}$. The samples were thermally treated at $90^{\circ} \mathrm{C}$ for 30 minutes in air to remove solvent by evaporation.

A spectroscopic ellipsometer (UVISEL SE, Horiba-Jobin Yvon) was used to measure film thicknesses using a Cauchy equation to model the refractive index. Three measurements were made on each sample to check the sample surface thickness homogeneity, found to be in the $95 \%$ range.

Ellipsometry measurements of the glass transition temperature $T_{g}$ of the thin PMMA films were performed using a M2000 ellipsometer (Woollam) equipped with an Instec heat cell. Temperature scans between $20^{\circ} \mathrm{C}$ to $150^{\circ} \mathrm{C}$ were carried out at a $5^{\circ} \mathrm{C} / \mathrm{min}$ rate while the refractive index of the film was continuously monitored. Below and above $T_{g}$, the refractive index was found to vary linearly with temperature with different slopes (results not shown). The glass transition temperature was identified from the intercept of these two linear relationships.

In addition, glass transition temperature measurements of the bulk PMMA polymers were carried out by Differential Scanning Calorimetry (DSC) at the same heating rate using a Q200 (TA Instruments) apparatus. 


\section{Depth profiling}

Dual beam depth profiling has been performed on a ToF-SIMS instrument (ToF-SIMS 5, IONTOF GmbH, Münster, Germany). The sputtering has been performed using $\mathrm{Cs}^{+}$ion sources with energies ranging from 250 to $1000 \mathrm{eV}$ rastered over a $400 \times 400 \mu \mathrm{m}^{2}$ area.

The negative secondary ions mass spectra collection, from a $80 \times 80 \mu \mathrm{m}^{2}$ area centred in the middle of the sputtered crater, has been performed with $(15 \mathrm{keV})$ bismuth ions $\mathrm{Bi}_{3}{ }^{+}$, from negative secondary ions.

One challenge of ToF-SIMS polymer analysis is the charge accumulation at the sputtering interface resulting in changes in the energy distribution of the emitted secondary ions which degrades the mass resolution ${ }^{18}$. Taking into account the polymer insulating electrical properties, charge compensation, using a $20 \mathrm{~V}$ electron flood gun, was applied for all depth profiling measurements. The acquisition was made in the non-interlaced mode with a 1 second pause, in order to properly compensate the charges.

\section{Results and Discussion}

Negative ions acquisition mode has been chosen since Cs enhances highly negative ionization (and depress positive ionization); in addition the presence of oxygen, as an electronegative species prone to accept electrons, in the PMMA backbone will favour the formation of significant negative ions ${ }^{19}$. The ToF-SIMS depth profiles of the PMMA thin films for two molecular weights $(M w=20 \mathrm{~kg} / \mathrm{mol}$ and $100 \mathrm{~kg} / \mathrm{mol})$ obtained with $500 \mathrm{eV} \mathrm{Cs}^{+}$are shown in Figure. 1, where some of the characteristic fragment ion signals are represented as function of the sputtering time.

A transient region, due to the Cs built up at the surface ${ }^{12}$, is present during the early stages of both depth profiles and followed by an equilibrium regime where the intensity of the signal is nearly constant as a function of the sputtering time. Reflecting the polymer chemistry, the selected PMMA fragments $\mathrm{CHO}_{2}{ }^{-}$ at $m / z=45, \mathrm{C}_{3} \mathrm{H}_{3} \mathrm{O}^{-}$at $m / z=55, \mathrm{C}_{4} \mathrm{H}_{3} \mathrm{O}^{-}$at $m / z=67$ and $\mathrm{C}_{5} \mathrm{H}_{5}{ }^{-}$at $m / z=65$, are comparatively stable and their intensities logically start to decrease when reaching the Si substrate. The stability of the characteristic signals in the beginning of the depth profiles implies a limited change of the polymer chemistry and chemical damage. This is consistent with a previous study by Houssiau et $a l^{12,20}$ which successfully performed molecular depth profiling of PMMA using $\mathrm{Cs}^{+}$sputtering primary ions while preserving the fingerprint ions intensity in the spectrum.

For a given film thickness $(100 \mathrm{~nm})$, the time needed to reach the interface (measured as the time required to reach $50 \%$ of the total ${ }^{28} \mathrm{Si}$ signal) is different for both polymers (194 s for PMMA $100 \mathrm{~kg} / \mathrm{mol}$ and 138 s for PMMA $20 \mathrm{~kg} / \mathrm{mol}$ ) which suggests a molecular weight dependent sputter rate. In order to further 
evaluate the dependence of sputtering yields on molecular weight, the sputtering yield volume per primary ion (PI) expressed in $\mathrm{nm}^{3} / \mathrm{PI}$ has been determined for the different samples. This parameter has been calculated from the sputter time (time required to reach $50 \%$ of the total $\mathrm{Si}^{-}$signal), the film thickness, the sputtering analysis current and the sputtered area.

In Figure. 2, the sputtering yield $(Y)$ volumes of two sets of PMMA thin films differing in their thicknesses and sputtered by $\mathrm{Cs}^{+} 500 \mathrm{eV}$, are presented as functions of logarithm of the molecular weight $M_{w}$. For film thicknesses in the range $90-120 \mathrm{~nm}, Y$ decreases with increasing molecular weight until a nearly constant value is achieved for $M_{w}$ above about $\sim 32 \mathrm{~kg} / \mathrm{mol}$. For thicknesses in the range 120-180 nm, the existence of a plateau region is less evident but there is a clear indication of a decreased sensitivity of $Y$ on $M_{w}$ above the $32 \mathrm{~kg} / \mathrm{mol}$ threshold.

Although the energy deposited on the surface per projectile ion is in the range of $500 \mathrm{eV}$, much higher than the intermolecular binding energies which generates fragmentation ${ }^{21}$, the sensitivity of the sputtering yield to the chemical nature of the analysed material is preserved. As a matter of fact, for lower molecular masses with higher end-groups densities and shorter backbone monomer chains, $Y$ is higher than for polymers with longer and heavier backbone chains. As stated by Seah $e t a l^{8}$, the difference in the number of end-groups per unit volume between polymers with different molecular weights could explain the dependence of sputtering yield on the chemical structure of the PMMA films. Working on Cristaudo et $a l,{ }^{4}$ data, Seah et $a l^{78}$ postulated that, when using polyatomic argon cluster ions to sputter polymer thin films, the increased number of end groups enhances the sputtering yields ${ }^{7,8}$. Indeed, when the molecular weight increases the number of end-groups decreases.

An alternate discussion of these molecular weight effects was provided by Cristaudo $e \mathrm{al}^{4}$ who postulated that the known increase in the glass transition temperature of the polymers with their molecular weights ${ }^{22}$ could also account for the observed decrease in the sputtering yield with $M_{w}$. Indeed, $T_{g}$ is a notably important physical property which governs the mobility of polymer chains. It is known to be essentially independent of the $M_{w}$, except for the region of low degrees of polymerization, where $T_{g}$ increases with increasing $M_{w}{ }^{23-25}$. The enhanced sputtering yield at low $M_{w}$ could therefore be attributed to the enhanced translational mobility of the polymer chains as a result of their depressed $T_{g}$.

In order to evaluate the relevance of such an hypothesis for our polymer films, while taking into account nano-confinement effects which are known to shift the glass transition temperature of thin films from bulk values $^{26,27}$, ellipsometry measurements of the glass transition temperature of the thin films have been performed as function of the molecular weight (Figure 3). In addition, $T_{g}$ data of the investigated bulk 
PMMA polymers as measured by Differential Scanning Calorimetry (DSC) at the same heating rate are reported in Figure 3. In the range 10-100 kg/mol, it appears that the $T_{g}$ of the thin films is slightly higher than that of bulk PMMA. Although the thickness-dependence of the $T_{g}$ of thin films is still being debated, such an increase could be attributed to nano-confinement effects as experimental values seem to be reliable for films thicker than $15 \mathrm{~nm}^{28}$.

The results presented in Figure. 3, indicate that $T_{g}$ of the $100 \mathrm{~nm}$ thick PMMA films under investigation are actually dependent on the $M_{w}$, increasing dramatically from the lowest molecular weight to reach a steady value around a $M_{w}$ of $\sim 20 \mathrm{~kg} / \mathrm{mol}$. It should be noted that the measured values are slightly superior to the $T_{g}$ of a bulk PMMA of infinite molecular weight $\left(\sim 108^{\circ} \mathrm{C}\right)^{29,30}$, consistently with the effect of nanoconfinement which are expected to limit the chain mobility, thus displacing the glass transition towards higher values ${ }^{26}$.

In the present ToF-SIMS experiments, the observed molecular weight dependence of the sputtering yield could be accounted for by the enhanced mobility of the polymer chain only if the surface temperature of the films is increased locally above $T_{g}$ by the ion beam. Although an accurate measurement of the local film surface temperature during sputtering is a difficult task, the occurrence of such $T_{g}$ effects was indirectly assessed from measurements of the sputtering yield as a function of temperature. In Figure. 4, the sputtering yield volumes of PMMA $20 \mathrm{~kg} / \mathrm{mol}$ and $100 \mathrm{~kg} / \mathrm{mol}$ sputtered by $500 \mathrm{eV}$ caesium ions at different temperatures are presented. It appears that the sputtering yield is nearly independent of temperature until a threshold value $\left(96^{\circ} \mathrm{C}\right.$ for $20 \mathrm{~kg} / \mathrm{mol}$ and $100^{\circ} \mathrm{C}$ for $\left.100 \mathrm{~kg} / \mathrm{mol}\right)$ which is close to the measured $T_{g}$ of the films under consideration. Above this threshold temperature, $Y$ increases dramatically, probably as a result of enhanced free radical formation and chain mobility in the glass transition zone. Both effects are likely to enhance the depolymerisation mechanisms and boost material removal.

These results clearly demonstrate that glass transition effects are not involved in sputtering experiments carried out at room temperature. In other words, film temperature remains far below $T_{g}$ during experiments at room temperature and the dependence of the sputtering yield on $M_{w}$ can barely be accounted for by the molecular weight dependent $T_{g}$ of the films. Instead, a dependency of the sputtering yield on the molecular weight between entanglements $\left(M_{e}\right)$ could be considered. Amorphous polymer chains in the solid or melted states are known to be entangled as a result of the ability of the macromolecules to adopt randomcoil conformations ${ }^{31}$. The so-called molecular weight between entanglements- $M_{e}$ is defined as the average molar mass of the polymer segment in between two consecutive entanglements points. Below $M_{e}$, polymer chains are un-entangled and the polymer materials presents physical and mechanical properties which are drastically different form the entangled state. $M_{e}$ is known to be dependent on the polymer's chemical 
structure. For PMMA, the entanglement molecular weight $M_{e}$ is around $11 \mathrm{~kg} / \mathrm{mol}^{31}$, a value close to the critical molecular weight above which the sensitivity of $Y$ on $M_{w}$ was observed to decrease (Figure. 2). The enhancement of $Y$ below $M_{e}$ could therefore tentatively be attributed to an easier depolymerisation of the un-entangled polymer chains.

The measured decay of $Y$ as a function of the molecular weight using monoatomic ion sputtering is consistent with the results obtained using argon clusters on PMMA thin films ${ }^{4}$. However, the magnitude of the yields achieved when using monoatomic ions ( $Y$ varies from $0.4 \mathrm{~nm}^{3} / \mathrm{PI}$ to $0.75 \mathrm{~nm}^{3} / \mathrm{PI}$ ) is one hundred times smaller than the values obtained with argon cluster ions by Cristaudo et $a l^{4}$ ( $Y$ varies from $20 \mathrm{~nm}^{3} / \mathrm{PI}$ to $110 \mathrm{~nm}^{3} / \mathrm{PI}$ ). This can be explained by the difference in the sputtering mechanisms between monoatomic and polyatomic ions. On one hand, polyatomic ions bombard the target on a large scale with all the constitutive atoms penetrating collectively, thus constraining the resulting damage to a narrower volume and limiting the damage to the upper layers. On the other hand, monoatomic ions induce upon impact a collision cascade, penetrating farther in depth. In the case of monoatomic ions, the generation of large fragment ions along with oligomers requires that several cascades intersect and cross to produce high-efficient yield events, which is unlikely to happen ${ }^{10,16}$. According to molecular dynamics (MD) simulations ${ }^{17}$, the use of polyatomic ions enhances the material removal as a result of the low energy density deposited on the surface, which justifies that higher sputtering yields are achieved with argon cluster ions than with caesium ions.

\section{Effect of thickness on the mean sputtering yield}

As shown in Figure.2, the dependence of $Y$ on $M_{w}$ follows the same decreasing trend whatever the film thickness. However, the magnitude of the sputtering yield is observed to decrease for thicker films in the whole investigated $M_{w}$ range. Cristaudo et al ${ }^{32}$ have observed a similar thickness-dependence of the sputtering yield of PS and PMMA films on silicon wafers upon argon cluster ion bombardment. The observed thickness dependence of $Y$ for polymer-based thin films was explained by the authors using a phenomenological model where the polymer film is supposed to be composed of two superimposed layers with different sputtering yields: $(i)$ at distances from the substrate less than a critical value of a few nanometers, $Y$ is at the highest due to a maximal contribution to the sputtering of the primary energy being back reflected into the polymer layer by the polymer/Si interface ${ }^{14}$ (ii) above this critical thickness, $Y$ has the value which would be achieved with bulk polymers. As a result, the gradual increase in thickness of the film above the critical thickness mitigates the interface effects till its complete cancellation. Indeed, 
Molecular dynamics carried out by Reznik et $a l^{33}$ have shown that $Y$ becomes independent of the thickness when it is much larger than the penetration depth of the projectile. However, when the polymer is not confined anymore by a dense substrate, the sputtering yield becomes independent of the thickness ${ }^{32}$.

Considering a similar reasoning, we tried to investigate the nano-confinement effect when using caesium monoatomic ions to bombard PMMA $4 \mathrm{~kg} / \mathrm{mol}$ and $15 \mathrm{~kg} / \mathrm{mol}$ thin films. These two materials were selected because the strongest changes in sputtering yield of PMMA films were found for low molecular mass. Accordingly, they were supposed to the most appropriate systems to reveal film thinning effects. As shown in Figure. 5, whatever the primary beam energy used, the maximal sputtering yield is achieved for the lowest investigated film thickness $(20 \mathrm{~nm})$. Then $Y$ decreases with thickness until a constant value is achieved around $70 \mathrm{~nm}$ for PMMA $4 \mathrm{~kg} / \mathrm{mol}$ and $90 \mathrm{~nm}$ for PMMA $15 \mathrm{~kg} / \mathrm{mol}$. The threshold value for PMMA $4 \mathrm{~kg} / \mathrm{mol}$ is slightly lower than that obtained on the same polymer by Cristaudo et al $l^{32}$ using 10 $\mathrm{keV} A r_{3000}^{+}$. These results seem to indicate that, when using monoatomic ions, the thickness from which an effect can be observed in sputtering yield is slightly reduced. In other words, slightly thinner films can be addressed with Cs sputtering, in comparison to GCIB, before worrying about uncontrolled changes in sputtering yields. This difference is assumed to arise from the fact that we might expect a higher primary ions implantation in the $\mathrm{Si}$ substrate with monoatomic ions than with cluster ones, which limits the sputtering process for thinner films. These first results show a significant but limited difference between polymer thin films sputtering mechanisms using cluster or monoatomic ions. They clearly need to be completed with data for materials at higher molecular weights to have a clear overview of the dependence on molecular weight of the thickness-sputtering mechanisms relationship.

\section{Effect of $\mathrm{Cs}^{+}$ion energy}

In order to get insights into the dependence on $Y\left(M_{w}\right)$ relationship on primary beam energy, depth profiling of PMMA thin films (>120 nm in thicknesses) has been performed at different $\mathrm{Cs}^{+}$energies $(250 \mathrm{eV}, 500$ $\mathrm{eV}$ and $1000 \mathrm{eV})$.

The depth profiles of PMMA $\left(M_{w}=54 \mathrm{~kg} / \mathrm{mol}\right)$ thin films displayed in Figure. 6 show that there is a significant difference in the $\mathrm{C}_{3} \mathrm{H}_{3} \mathrm{O}^{-}$ion intensities depending on the beam energies. When profiling the polymer layer, the characteristic $\mathrm{C}_{3} \mathrm{H}_{3} \mathrm{O}^{-}$ion intensity is higher when reducing beam energy. Furthermore, the initial transient is more pronounced for higher energies. The transition between the $\mathrm{C}_{3} \mathrm{H}_{3} \mathrm{O}^{-}$and $\mathrm{Si}$ signals at the polymer/silicon interface is also narrower when the $\mathrm{Cs}^{+}$ion energy is increased.

When sputtering with $1 \mathrm{keV} \mathrm{Cs}^{+}$, the polymer characteristic ion signal is unstable and unable to reach a steady-state value during the course of the sputtering process. Previous studies attributed this behaviour 
to damage accumulation ${ }^{34,35}$ or ionisation ${ }^{36}$ effects depending on the nature of the primary ion source. A lowered level of implanted Cs has also to be considered leading to less reactions with free radicals and subsequently less neutralization of polymeric chain degradation. In addition, increasing energy deteriorates the depth profiles quality due to induced roughness at the bottom of the crater. Indeed, Atomic Force Microscopy (AFM) measurements over a $1 \mu \mathrm{m}^{2}$ area (not shown in this paper) indicated that the rms roughness was respectively $0.23 \mathrm{~nm}, 1.29 \mathrm{~nm}$ and $2.49 \mathrm{~nm}$ for the virgin PMMA film and the craters sputtered at $500 \mathrm{eV}$ and $1 \mathrm{keV}$. These changes were found to be independent on the molecular weight. The change in the sputtering yield volume with the primary beam energy for the different molecular weight films is shown in figure7. As expected, the overall sputtering yield increases with the energy of the ion beam, while the observed decrease in $Y$ with $M_{w}$ is preserved. In order to quantify these effects, experimental data were fitted to the following empirical equation initially developed for argon sputtering ${ }^{5,8}$

$$
Y=Y_{0}+\frac{\alpha}{M w^{n}}
$$

Where $Y_{O}$ is the sputtering yield of a PMMA polymer of infinite molecular weight, $n$ is an empirical parameter quantifying the dependence of the sputtering yield on the molecular weight and $\alpha$ is a constant. The values of the fitting parameters $n$ (Table.2) indicates that the sensitivity of the sputtering yield to $M_{w}$ is enhanced at $1 \mathrm{keV}$, which could reflect a change in the sputtering mechanisms as compared to 250 and $500 \mathrm{eV}$.

In figure.8, the sputtering yield volume has been reported as a function of the logarithm of the primary ion beam energy. Whatever the molecular weight, this plot tends to suggest the existence on an energy threshold below which $Y$ vanishes. The value of this threshold and its dependency on the molecular weight would, however, requires further investigations.

\section{Conclusions}

In this work, we investigated the specificity of caesium monoatomic ions when it comes to organic materials depth profiling in terms of sensitivity to structural parameters, specifically the molecular weight. Actually, a dependence of caesium sputtering yields on molecular weight was evidenced with some similarities with cluster ions sputtering ${ }^{4}$. Indeed, it decreases with increasing molecular weight until a threshold $\mathrm{Mw}$ value around $(32 \mathrm{~kg} / \mathrm{mol})$ which is slightly higher than the one obtained with argon clusters (20 kg/mol). However, compared to previous studies of PMMA with argon clusters the sputtering yields are 10 to 100 times smaller which is potentially advantageous by giving suitable period of time for the depth profiling of very thin polymer multilayers. 
In addition, from an investigation of the temperature-dependence of the sputtering yield, we have concluded that these dependence of the sputtering yield on molecular weight relationship at room temperature cannot be related to the $M_{w}$ dependence of the PMMA glass transition temperature. Instead, the hypothesis is that the sputtering yield is enhanced below the entanglement molecular weight. It was also evidenced that the ion beam energy doesn't change the overall way the sputtering yields varies with $M_{w}$. However, an explicit logarithmic dependence of $Y$ on beam energy has been evidenced with parameters slightly dependent on the molecular weight. The variation of $Y$ with the primary ion energy not only indicates changes in the ion-solid interactions at higher energies but also reveals the existence of a threshold energy below which sputtering seems to cease. This threshold, below which sputtering would no longer be possible, is found to be slightly dependent on the chemical structure of the polymer. Furthermore, the fitting of the variations of $Y$ with $M_{w}$, reveals a sensitivity to the molecular weight of greater magnitude at higher energies.

Finally, the influence of the thickness of the studied film has been explored. It provided relevant elements in qualifying the differences in polymer sputtering mechanisms between monoatomic and cluster ions. This preliminary study, performed on 4 and $15 \mathrm{~kg} / \mathrm{mol}$ PMMA films put into evidence a nano-confinement effect less pronounced than the one observed when using argon clusters ${ }^{32}$. The intensity of the phenomenon is much lower and begins for thinner layers than recorded with argon clusters. And the effect seems especially weak at low $\mathrm{Cs}^{+}$energy $(250 \mathrm{eV})$ where it was seen to be limited to a doubling of sputtering yields. It's worth nothing this observation since it makes in regard to GCIB, low energy caesium sputtering promising for depth profiling of very thin polymeric layers on dense substrates. However these results have to be generalized and completed with further investigations for higher molecular masses, other types of polymers in order to confirm, potentially, the limited nano-confinement effect when using monoatomic ions compared to polyatomic ions.

\section{Acknowledgment}

This work was performed on the PlatForm for NanoCharacterisation (PFNC) of CEA-Leti and was supported by the "Recherche Technologique de Base" Program of the French Ministry of Research. 


\section{References}

1. Chan C-M, Weng LT, Lau Y-TR. Polymer surface structures determined using ToF-SIMS. Rev Anal Chem. 2014;33:11-30. doi:10.1515/revac-2013-0015

2. Zhou C, Sun D, Garcia R, Stevie FA. Determination of chemical composition in multilayer polymer film using ToF-SIMS. Anal Methods. 2018;10(21):2444-2449.

doi:10.1039/C8AY00344K

3. Ninomiya S, Ichiki K, Yamada H, et al. Precise and fast secondary ion mass spectrometry depth profiling of polymer materials with large Ar cluster ion beams. Rapid Commun Mass Spectrom. 2009;23(11):1601-1606. doi:10.1002/rcm.4046

4. Cristaudo V, Poleunis C, Czerwinski B, Delcorte A. Ar cluster sputtering of polymers: effects of cluster size and molecular weights: Ar cluster sputtering of polymers. Surf Interface Anal. 2014;46(S1):79-82. doi:10.1002/sia.5424

5. Cheng J, Wucher A, Winograd N. Molecular Depth Profiling with Cluster Ion Beams. J Phys Chem B. 2006;110(16):8329-8336. doi:10.1021/jp0573341

6. Delcorte A, Debongnie M. Macromolecular Sample Sputtering by Large Ar and CH4 Clusters: Elucidating Chain Size and Projectile Effects with Molecular Dynamics. J Phys Chem C. 2015;119(46):25868-25879. doi:10.1021/acs.jpcc.5b07007

7. Seah MP. Argon cluster size-dependence of sputtering yields of polymers: molecular weights and the universal equation: Ar GCIB sputtering and the universal equation. Surf Interface Anal. 2015;47(1):169-172. doi:10.1002/sia.5656

8. Seah MP. Universal Equation for Argon Gas Cluster Sputtering Yields. J Phys Chem C. 2013;117(24):12622-12632. doi:10.1021/jp402684c

9. Rabbani S, Barber AM, Fletcher JS, Lockyer NP, Vickerman JC. TOF-SIMS with Argon Gas Cluster Ion Beams: A Comparison with C60+. Anal Chem. 2011;83(10):3793-3800. doi:10.1021/ac200288v

10. Delcorte A, Bertrand P, Garrison BJ. Collision Cascade and Sputtering Process in a Polymer. $J$ Phys Chem B. 2001;105(39):9474-9486. doi:10.1021/jp011099e

11. Colla TJ, Aderjan R, Kissel R, Urbassek HM. Sputtering of Au (111) induced by 16-keV Au cluster bombardment: Spikes, craters, late emission, and fluctuations. Phys Rev B. 2000;62(12):8487-8493. doi:10.1103/PhysRevB.62.8487

12. Mine N, Douhard B, Brison J, Houssiau L. Molecular depth-profiling of polycarbonate with lowenergy Cs+ ions. Rapid Commun Mass Spectrom. 2007;21(16):2680-2684. doi:10.1002/rcm.3135

13. Houssiau L, Mine N. Molecular depth profiling of polymers with very low energy reactive ions. Surf Interface Anal. 2010;42(8):1402-1408. doi:10.1002/sia.3159 
14. Matsunami N, Yamamura Y, Itikawa Y, Itoh N, Kazumata Y, Miyagawa S, Morita K, Shimizu R, Tawara H. Energy dependence of the ion-induced sputtering yields of monatomic solids. At Data Nucl Data Tables. 1984;31(1):1-80. doi:10.1016/0092-640X(84)90016-0

15. Rading D, Moellers R, Cramer H-G, Niehuis E. Dual beam depth profiling of polymer materials: comparison of C 60 and Ar cluster ion beams for sputtering: Dual beam depth profiling of polymer materials. Surf Interface Anal. 2013;45(1):171-174. doi:10.1002/sia.5122

16. Delcorte A, Garrison BJ. High Yield Events of Molecular Emission Induced by Kiloelectronvolt Particle Bombardment. J Phys Chem B. 2000;104(29):6785-6800. doi:10.1021/jp001374h

17. Delcorte A, Garrison BJ, Hamraoui K. Dynamics of Molecular Impacts on Soft Materials: From Fullerenes to Organic Nanodrops. Anal Chem. 2009;81(16):6676-6686. doi:10.1021/ac900746x

18. Wang Z, Liu B, Zhao EW, et al. Argon Cluster Sputtering Source for ToF-SIMS Depth Profiling of Insulating Materials: High Sputter Rate and Accurate Interfacial Information. J Am Soc Mass Spectrom. 2015;26(8):1283-1290. doi:10.1007/s13361-015-1159-1

19. Piwowar AM, Vickerman JC. The role of molecular weight on the ToF-SIMS spectra of PMMA using Au+ and C60+ primary ions. Surf Interface Anal. 2010;42(8):1387-1392. doi:10.1002/sia.3197

20. Houssiau L, Douhard B, Mine N. Molecular depth profiling of polymers with very low energy ions. Appl Surf Sci. 2008;255(4):970-972. doi:10.1016/j.apsusc.2008.05.027

21. Wehbe N, Pireaux J-J, Houssiau L. XPS Evidence for Negative Ion Formation in SIMS Depth Profiling of Organic Material with Cesium. J Phys Chem C. 2014;118(46):26613-26620. doi:10.1021/jp501851f

22. Dibenedetto AT, Dilandro L. Correlation of glass transition temperature and molecular weight: A model based on the principle of corresponding states. J Polym Sci Part B Polym Phys. 1989;27(7):1405-1417. doi:10.1002/polb.1989.090270703

23. Turner DT. Glass transition elevation by polymer entanglements. Polymer. 1978;19(7):789-796. doi:10.1016/0032-3861(78)90006-X

24. Thompson EV. Dependence of the glass transition temperature of poly(methyl methacrylate) on tacticity and molecular weight. J Polym Sci Part -2 Polym Phys. 1966;4(2):199-208. doi:10.1002/pol.1966.160040204

25. Beevers RB, White EFT. Physical properties of vinyl polymers. Part 1.-Dependence of the glasstransition temperature of polymethylmethacrylate on molecular weight. Trans Faraday Soc. 1960;56(0):744-752. doi:10.1039/TF9605600744

26. Singh L, Ludovice PJ, Henderson CL. Influence of molecular weight and film thickness on the glass transition temperature and coefficient of thermal expansion of supported ultrathin polymer films. Thin Solid Films. 2004;449(1):231-241. doi:10.1016/S0040-6090(03)01353-1

27. Keddie JL, Jones RAL, Cory RA. Size-Dependent Depression of the Glass Transition Temperature in Polymer Films. Europhys Lett EPL. 1994;27(1):59-64. doi:10.1209/0295-5075/27/1/011 
28. Hinrichs K, Eichhorn K-J. Ellipsometry of Functional Organic Surfaces and Films.; Springer Series in Surface Sciences, Springer, 2018.

29. Porter CE, Blum FD. Thermal Characterization of PMMA Thin Films Using Modulated Differential Scanning Calorimetry. Macromolecules. 2000;33(19):7016-7020. doi:10.1021/ma0003021

30. Sokolov AP, Novikov VN, Ding Y. Why many polymers are so fragile. J Phys Condens Matter. 2007;19(20):205116. doi:10.1088/0953-8984/19/20/205116

31. Wool RP. Polymer entanglements. Macromolecules. 1993;26(7):1564-1569. doi:10.1021/ma00059a012

32. Cristaudo V, Poleunis C, Delcorte A. Effect of nanoconfinement on the sputter yield in ultrathin polymeric films: Experiments and model. Appl Surf Sci. 2018;444:780-788. doi:10.1016/j.apsusc.2018.03.041

33. Rzeznik L, Czerwinski B, Garrison BJ, Winograd N, Postawa Z. Microscopic Insight into the Sputtering of Thin Polystyrene Films on Ag $\{111\}$ Induced by Large and Slow Ar Clusters. J Phys Chem C. 2008;112(2):521-531. doi:10.1021/jp076667q

34. Fleischmann C, Conard T, Havelund R, et al. Fundamental aspects of Ar $\mathrm{n}+$ SIMS profiling of common organic semiconductors: Organic depth profiling. Surf Interface Anal. 2014;46(S1):5457. doi:10.1002/sia.5621

35. Wang S-K, Chang H-Y, Chu Y-H, et al. Effect of energy per atom ( E / n ) on the Ar gas cluster ion beam (Ar-GCIB) and O 2 + cosputter process. The Analyst. 2019;144(10):3323-3333. doi:10.1039/C8AN02452A

36. Cramer H-G, Grehl T, Kollmer F, Moellers R, Niehuis E, Rading D. Depth profiling of organic materials using improved ion beam conditions. Proc Sixt Int Conf Second Ion Mass Spectrom SIMS XVI. 2008;255(4):966-969. doi:10.1016/j.apsusc.2008.05.028 


\section{Tables}

Table 1. Molecular characteristics of the studied PMMA polymers

\begin{tabular}{|c|l|c|}
\hline $\boldsymbol{M}_{\boldsymbol{w}}(\mathbf{k g} / \mathbf{m o l})^{\mathbf{a}}$ & $\boldsymbol{I}_{\boldsymbol{p}}^{\mathbf{b}}$ & Supplying source \\
\hline 1260 & 1.16 & Polymer Source \\
\hline $356^{*}$ & 1.4 & Sigma Aldrich \\
\hline $107^{*}$ & 1.05 & Sigma Aldrich \\
\hline 54 & 1.09 & Polymer Source \\
\hline 32 & 1.09 & Polymer Source \\
\hline $15^{*}$ & 1.02 & Sigma Aldrich \\
\hline $7.5^{*}$ & 1.04 & Sigma Aldrich \\
\hline $4.2^{*}$ & 1.7 & Sigma Aldrich \\
\hline
\end{tabular}

${ }^{\mathrm{a}} M_{w}$ : Mass average molar mass

${ }^{\mathrm{b}} I_{p}$ : Polydispersity index $=M_{w} / M_{n}$

*: Polydispersity measured by Steric Exclusion Chromatography (SEC)

Table 2. Values of the fitting parameters of equation (1) giving the sputtering yield $Y$ as a function of the molecular weight $M_{w}$ for thin films with thicknesses in the range 120-180 nm.

\begin{tabular}{cccc}
\hline Energy $(\mathrm{eV})$ & $\boldsymbol{Y}_{\boldsymbol{0}}$ & $\boldsymbol{\alpha}$ & $\boldsymbol{n}$ \\
250 & 0.13 & 0.12 & 0.33 \\
500 & 0.48 & 0.27 & 0.33 \\
1000 & 0.78 & 0.42 & 0.45 \\
\hline
\end{tabular}




\section{Figures}
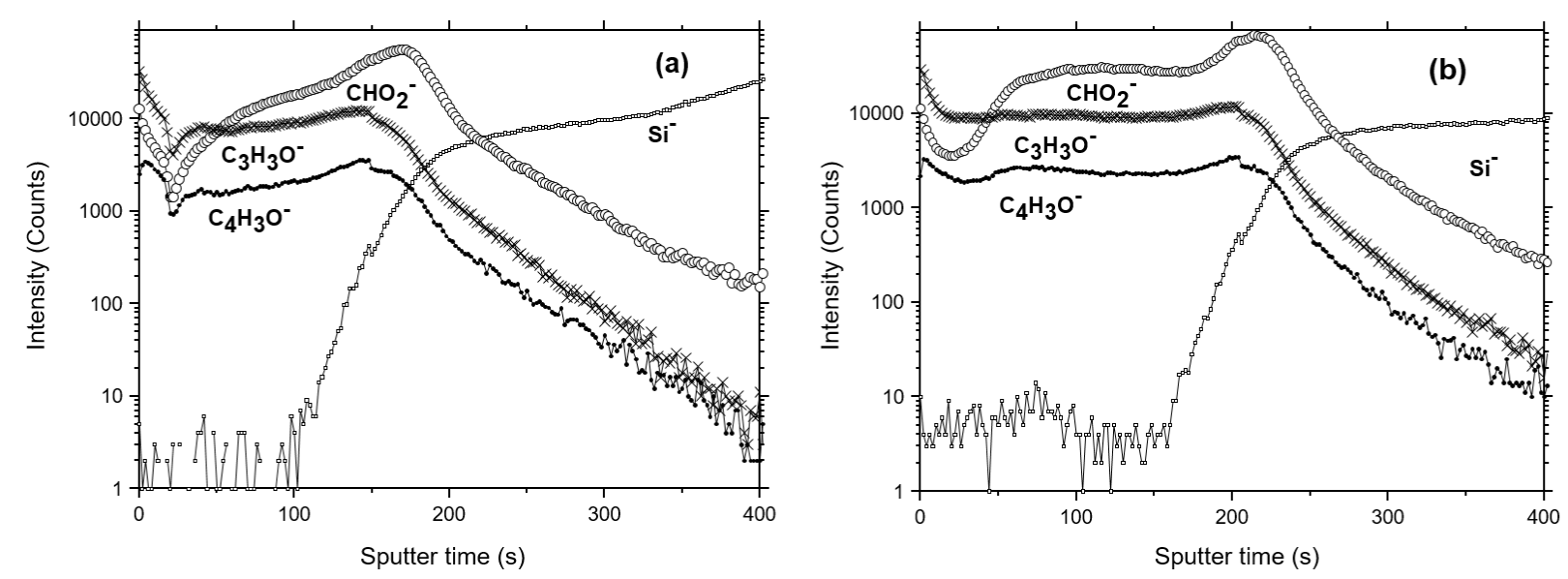

Figure 1. Negative ToF-SIMS depth profiles of PMMA (film thickness $100 \mathrm{~nm}$ ) with different molecular weights: (a) $20 \mathrm{~kg} / \mathrm{mol}$; (b) $100 \mathrm{~kg} / \mathrm{mol}$ (sputtering by $500 \mathrm{eV} \mathrm{Cs}^{+}$ions).

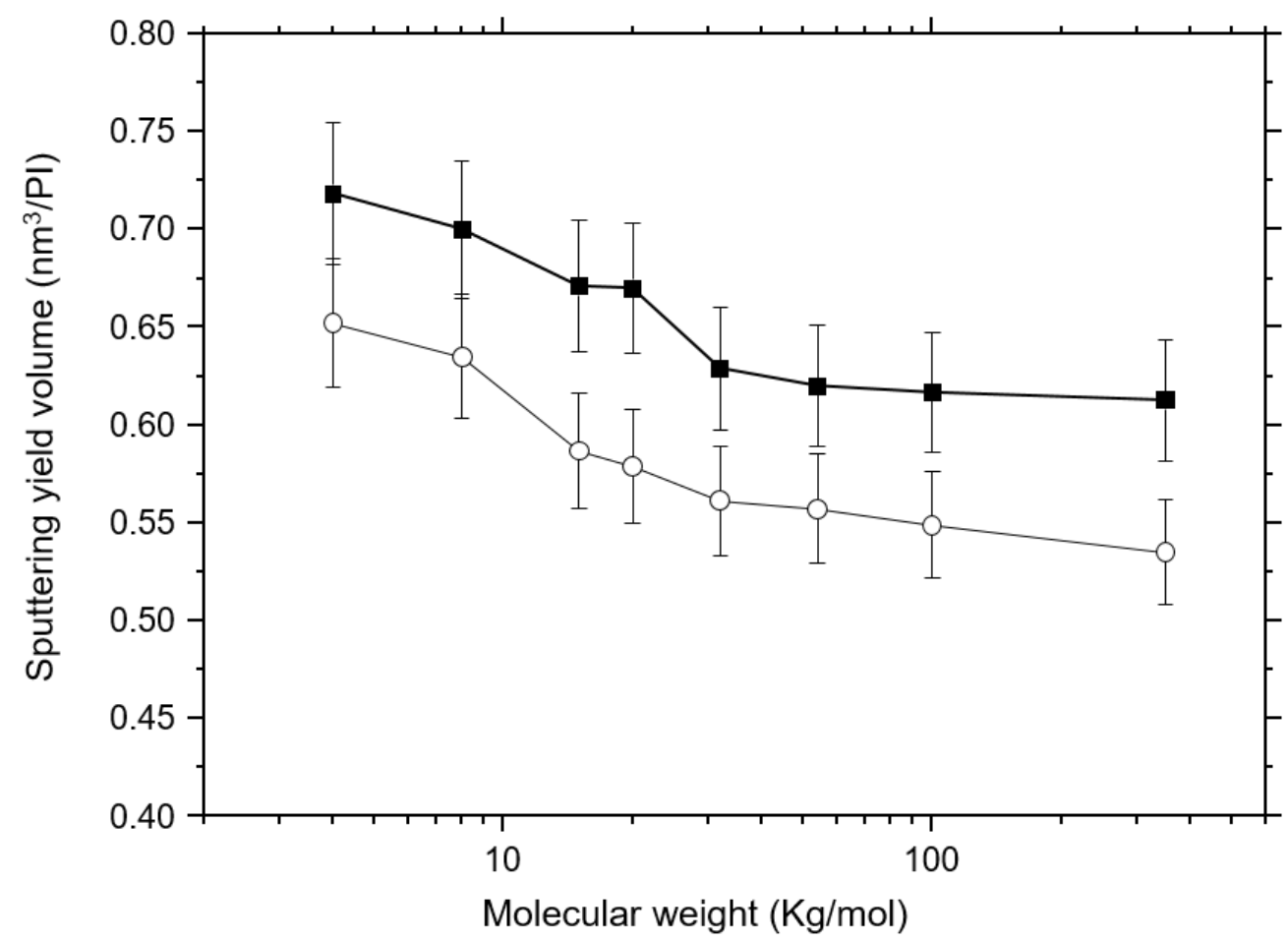

Figure 2. Sputtering yield volumes as function of molecular weight for two sets of PMMA thin films with different range of thickness :(匹) 90-120 nm; (O) 120-180 nm (500 eV Cs ${ }^{+}$ion sputtering). 


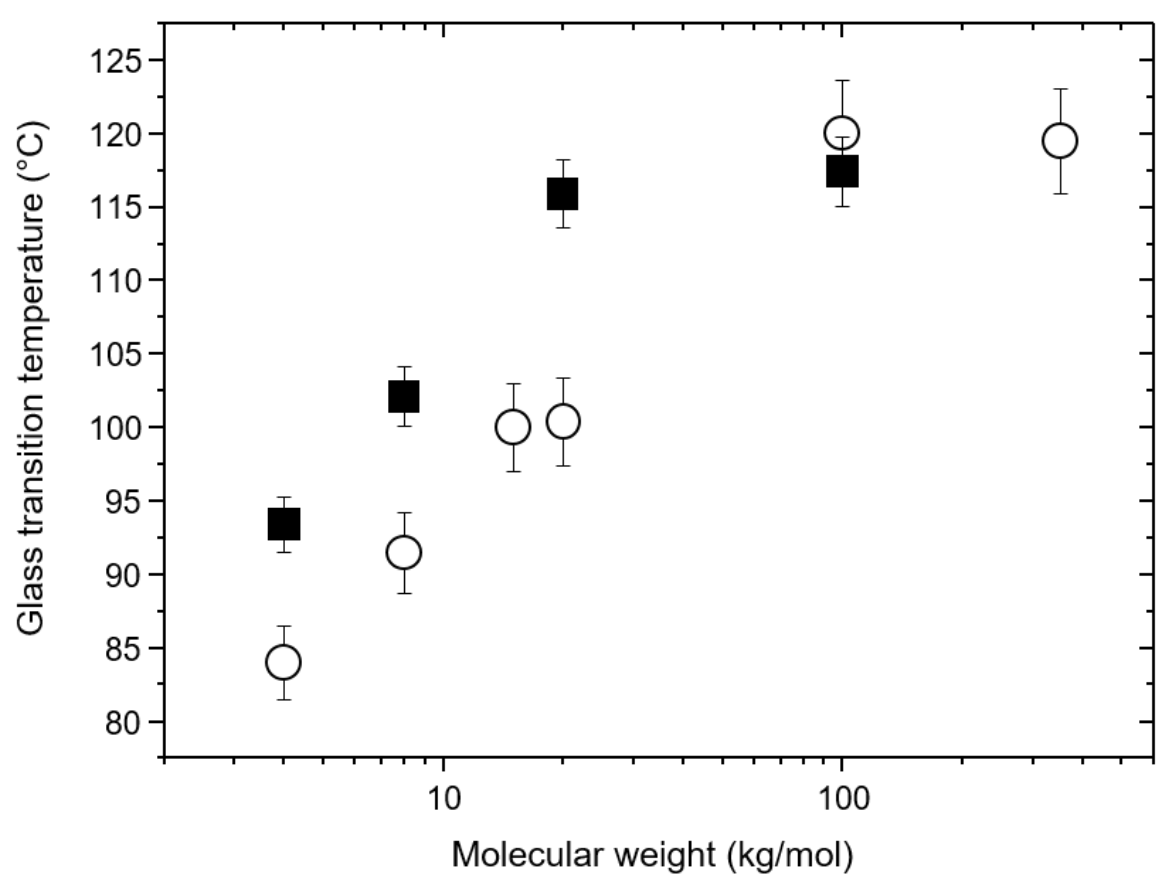

Figure 3. Glass transition temperatures of ( $)$ PMMA thin films (thickness: $100 \mathrm{~nm}$ ) and (O) bulk PMMA as a function of molecular weight, as measured by ellipsometry and DSC, respectively $\left(5^{\circ} \mathrm{C} / \mathrm{min}\right)$.

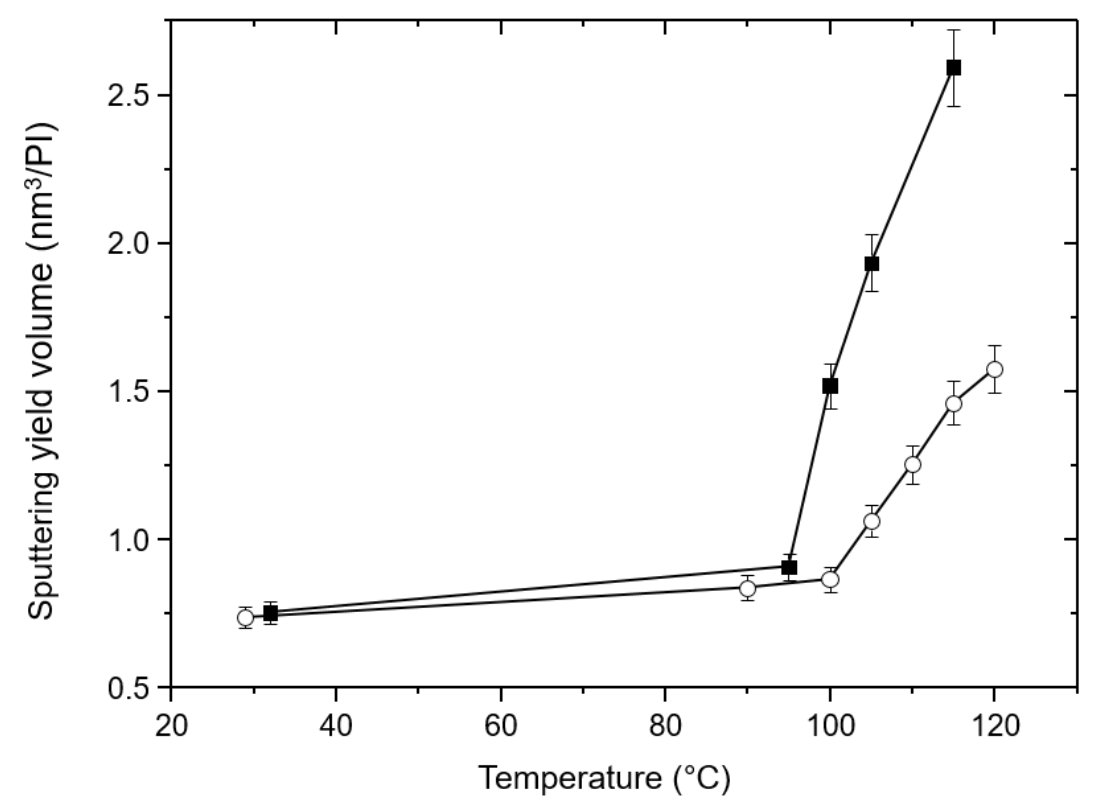

Figure 4. Sputtering yield volumes of PMMA thin films (ם) $20 \mathrm{~kg} / \mathrm{mol}$ and (O) $100 \mathrm{~kg} / \mathrm{mol}$ as a function of temperature (film thickness: $100 \mathrm{~nm}$ ). 

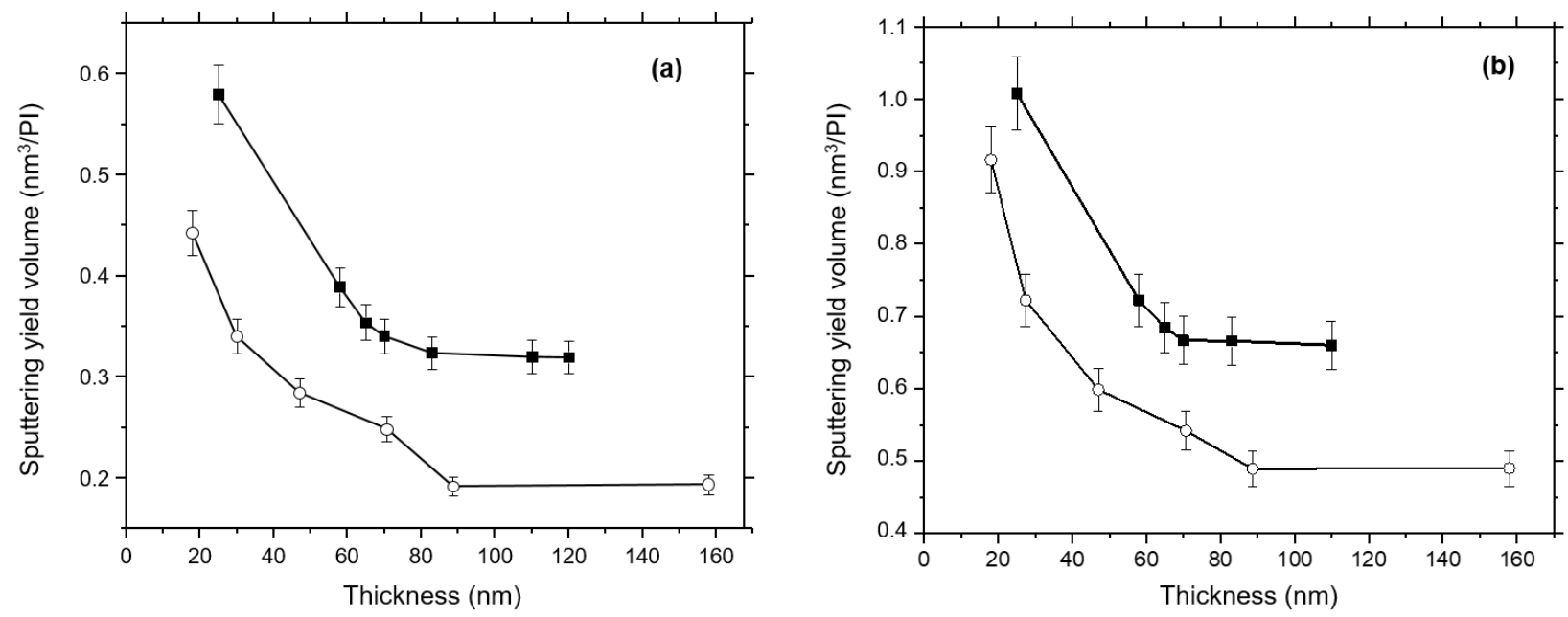

Figure 5. Sputtering yields of PMMA thin films ( 4 ) $4 \mathrm{~kg} / \mathrm{mol}$ and $(O) 15 \mathrm{~kg} / \mathrm{mol}$ sputtered by $\mathrm{Cs}^{+}$ions as function of film thickness at (a) $250 \mathrm{eV}$ and (b) $500 \mathrm{eV}$.
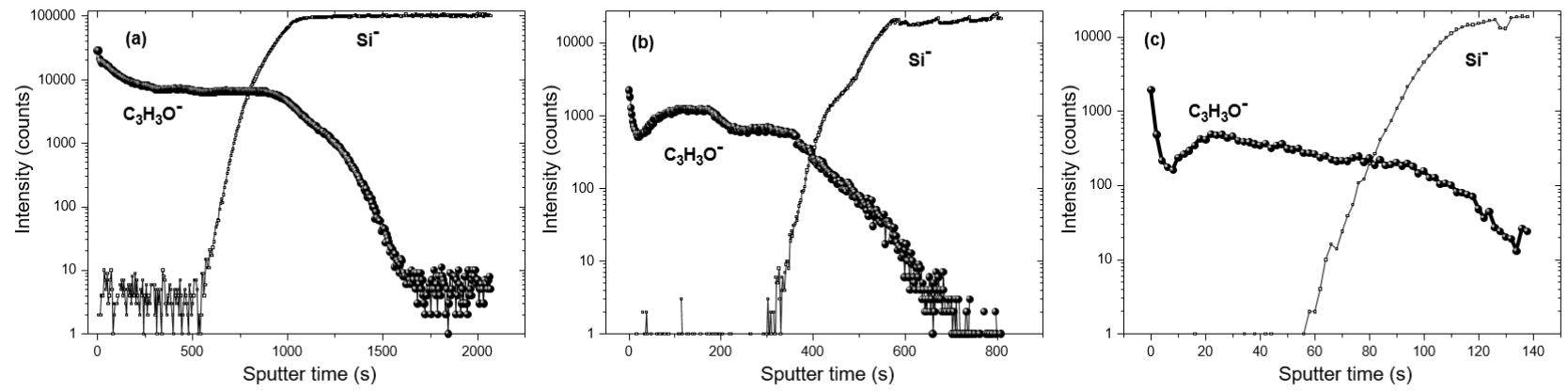

Figure 6. Depth profiles of $\mathrm{C}_{3} \mathrm{H}_{3} \mathrm{O}^{-}$and $\mathrm{Si}^{-}$ions of $120 \mathrm{~nm}$ PMMA thin films sputtered by $\mathrm{Cs}^{+}$ions at different beam energies: (a) $250 \mathrm{eV}$; (b) $500 \mathrm{eV}$; (c) $1000 \mathrm{eV}$. 


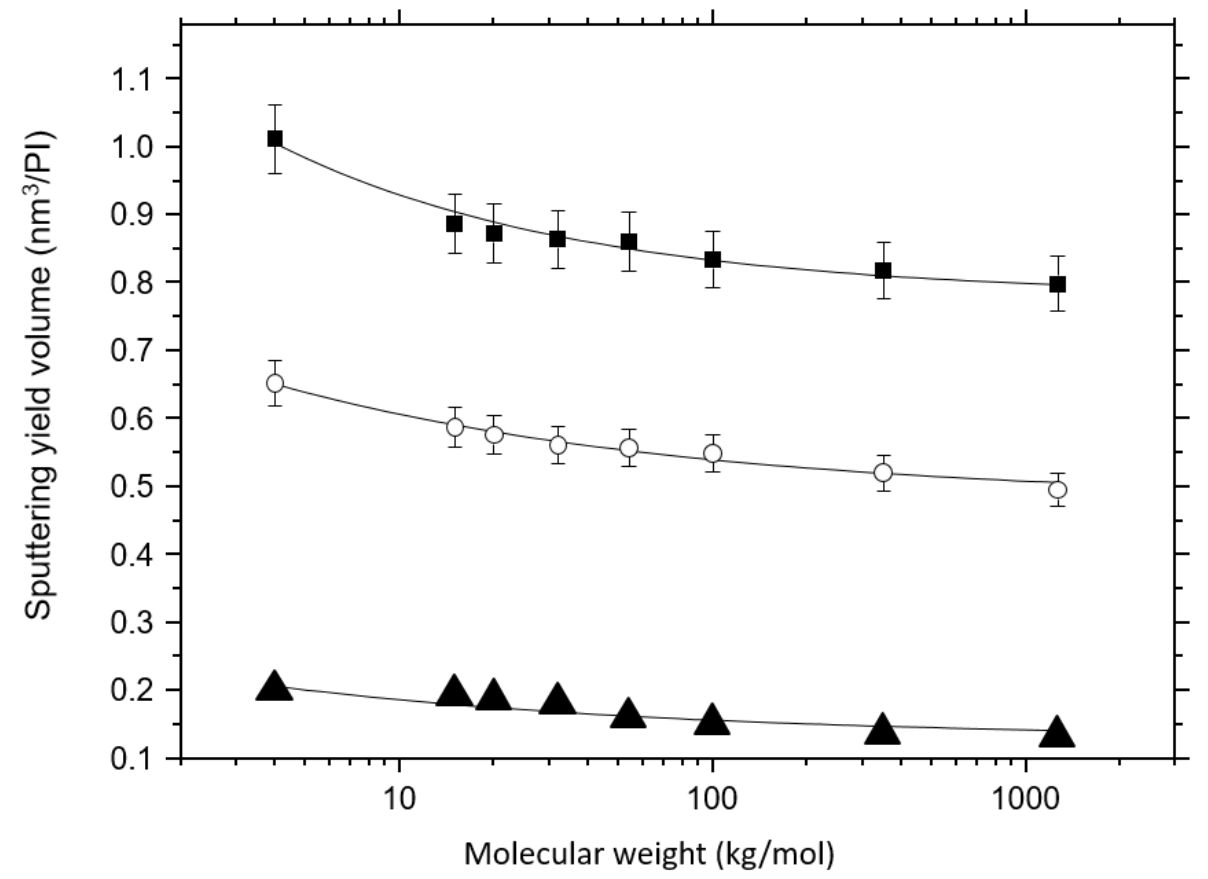

Figure 7. Sputtering yields of PMMA thin films as a function of molecular weight for different beam energies : (A) $250 \mathrm{eV}$; (O) $500 \mathrm{eV}$; (⿴) $1000 \mathrm{eV}$ (film thicknesses in the range 120-180 nm). Solids lines correspond to fits to equation (1).

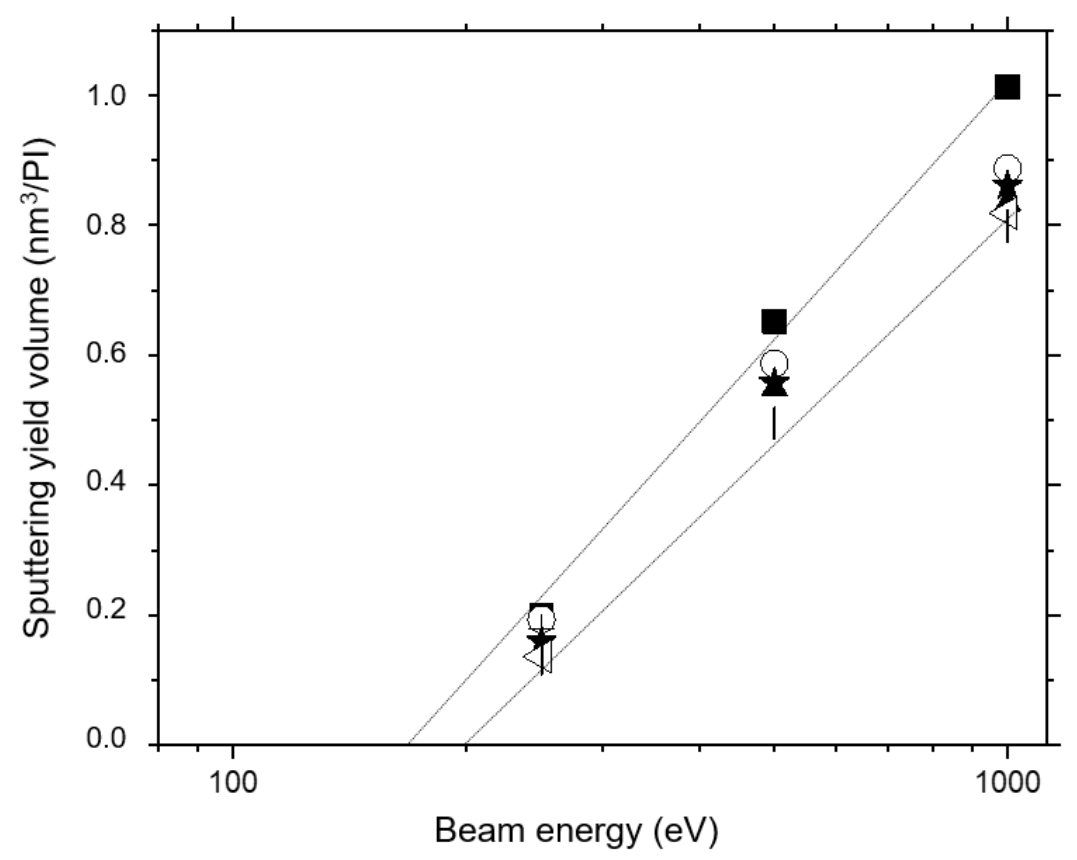

Figure 8. Sputtering yield volumes as a function of $\mathrm{Cs}^{+}$beam energy for PMMA thin films with different molecular weight $M_{w}$ (film thicknesses in the range 120-180 nm). (ロ) $4 \mathrm{~kg} / \mathrm{mol}$; (O) .

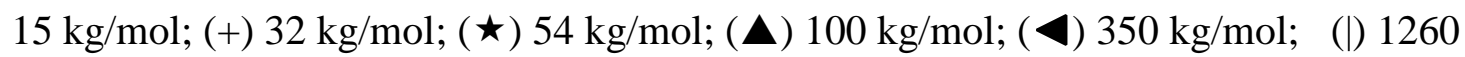
$\mathrm{kg} / \mathrm{mol}$. Dotted lines are guides for the eye. 